\title{
Short review on the diagnosis and treatment of bronchiectasis
}

Andrei Lesan, Alicia Elisabeth Lamle

PNEUMOLOGY

Pneumology Department, Iuliu Hatieganu University of Medicine and Pharmacy, Cluj-Napoca, Romania
DOI: $10.15386 /$ cjmed-1060

Manuscript received: 04.05.2018 Received in revised form: 11.07.2018 Accepted: 17.07.2018

Address for correspondence: alicia-lamle@hotmail.com

\begin{abstract}
Bronchiectasis refers to the permanent dilation of the bronchi. It is often a sequel of insufficiently treated lung disease that develops into a pathological pattern of dilated bronchi, which heightens susceptibility to further lung infections. Modernization of diagnostic procedures (computed tomography scan) and definition of a clinical picture (repeated lung infections with a chronic cough and persistent sputum production) have raised international awareness of the prevalence of the disease, leading to increasing interest in reviewing and renewing the treatment guidelines. We selectively conducted a research on PubMed using the following keywords: "bronchiectasis", "diagnosis", "treatment", "management", "antibiotics". This review focuses solely on bronchiectasis not due to cystic fibrosis. All relevant articles published after the year of 2000 were included.

The aim of this review is to provide an analytical update on the management of bronchiectasis, focusing on etiological factors as well as existing and developing treatment options for the disease.
\end{abstract}

Keywords: bronchiectasis, treatment, antibiotics, mucolytic, anti-inflammatory

\section{Introduction}

Bronchiectasis is defined as a permanent dilation of the bronchi. It seems to be a consequence of previous lung diseases, especially lung infections that were not treated sufficiently. This renders the lung tissue susceptible to further infections, as the mucociliary defense mechanism fails to regain its full ability to fight off infectious agents (a mechanism further described as the "vicious cycle theory"). Clinically, the disease manifests as recurrent episodes of respiratory tract infections together with an enduring cough and continuous production of sputum. To diagnose bronchiectasis, a high resolution computed tomography (HRCT) scan must be performed, evidencing persistent dilation of the airways. The treatment of bronchiectasis remains a challenge due to the multiple causes and facets of the disease. Studies emphasize the non-pharmacological treatment, including lifestyle modifications and physical therapy. Concomitantly, after identifying the underlying cause of the bronchial dilation, pharmacological therapy is aimed at the reduction of microbial invasion with antibiotics, as well as symptomatic relief with antiinflammatory drugs and mucolytic agents. Effective prevention measures such as smoking cessation and vaccines may prevent disease exacerbations. Nonetheless, the most important factor affecting the prognostic outcome remains the early detection, confirmed by a clinical diagnosis, to initiate a quick and effective treatment plan.

\section{Causes of bronchiectasis}

Bronchiectasis is said to be $25-50 \%$ idiopathic [1]. It has many etiologies and is often associated with other disorders. The most common causes for bronchiectasis include, in the following order: post-infection, cystic fibrosis (CF), immunodeficiency, chronic obstructive pulmonary disease (COPD), asthma, ciliary dyskinesia, and systemic diseases [2] (Table I). 
Table I. Causes of Bronchiectasis

$\begin{array}{ll} & \text { Bacteria: } \\ & \text { - Hemophilus influenza } \\ \text { Infectious } & \text { - Pseudomonas aeruginosa } \\ & \text { Atypical Aspergillus fumigatus } \\ & \text { Endobronchial obstruction } \\ & \text { - Enlarged lymph node } \\ & \text { - Carcinoid tumor } \\ & \text { Aspiration } \\ \text { - Gastric acid contents } \\ \text { - Foreign body aspiration } \\ \text { Systemic } \\ \text { - Celiac disease } \\ \text { - Yellow nail syndrome } \\ \text { Anatomic: } \\ \text { - Mounier-Kuhn Syndrome } \\ \text { - Williams-Campbell Syndrome } \\ \text { - Marfan Syndrome } \\ \text { - Relapsing polychondritis } \\ \text { Other: } \\ \text { - Cystic fibrosis } \\ \text { - Primary ciliary dyskinesia } \\ \text { - Kartagener Syndrome } \\ \text { - Hypogammaglobulinemia } \\ \end{array}$

\section{Pathogenesis}

Bronchiectasis is characterized by the permanent dilation of the bronchi, infection being the most common underlying cause of inflammation which, if left untreated, eventually leads to the dilation of the airway architecture [3]. To understand the pathogenesis of bronchiectasis, it is important to differentiate if the cause is truly infectious or if the patients develop infections due to an underlying predisposing condition [4].

Infectious and post-infectious causes of bronchiectasis

There are two different patterns occurring in bronchiectasis: current infectious diseases, where the lung is colonized by microorganisms, versus the occurrence of a post-infectious bronchiectasis, which develops in adults or children following a severe infection that may or may not have been sufficiently treated. In adults with post-infectious bronchiectasis, the mechanism seems to be a significant infection in early childhood, which causes structural damage to the developing lung and permits later bacterial infiltration that is inhibited in its proper clearance. Over time, a persistent infection may then result in bronchiectasis.

Although sputum production and impaired mucociliary clearance are persistent, bacterial colonization and markers of inflammation are intermittent in most patients [5]. The most common infectious agents are bacterial (Haemophilus influenza and Pseudomonas aeruginosa) and granulomatous (tuberculous and non-tuberculous mycobacteria) [6].

The destructive effect of chronic airway infections is described as the "vicious cycle theory" by Cole [7]. It describes how damaged airways become susceptible to chronic colonization, which merges into an inflammatory condition, which further results in injury and reduced resistance to infection (Figure I).

\section{NORMAL BRONCHECTASIS:VICIOUS CYCLE}

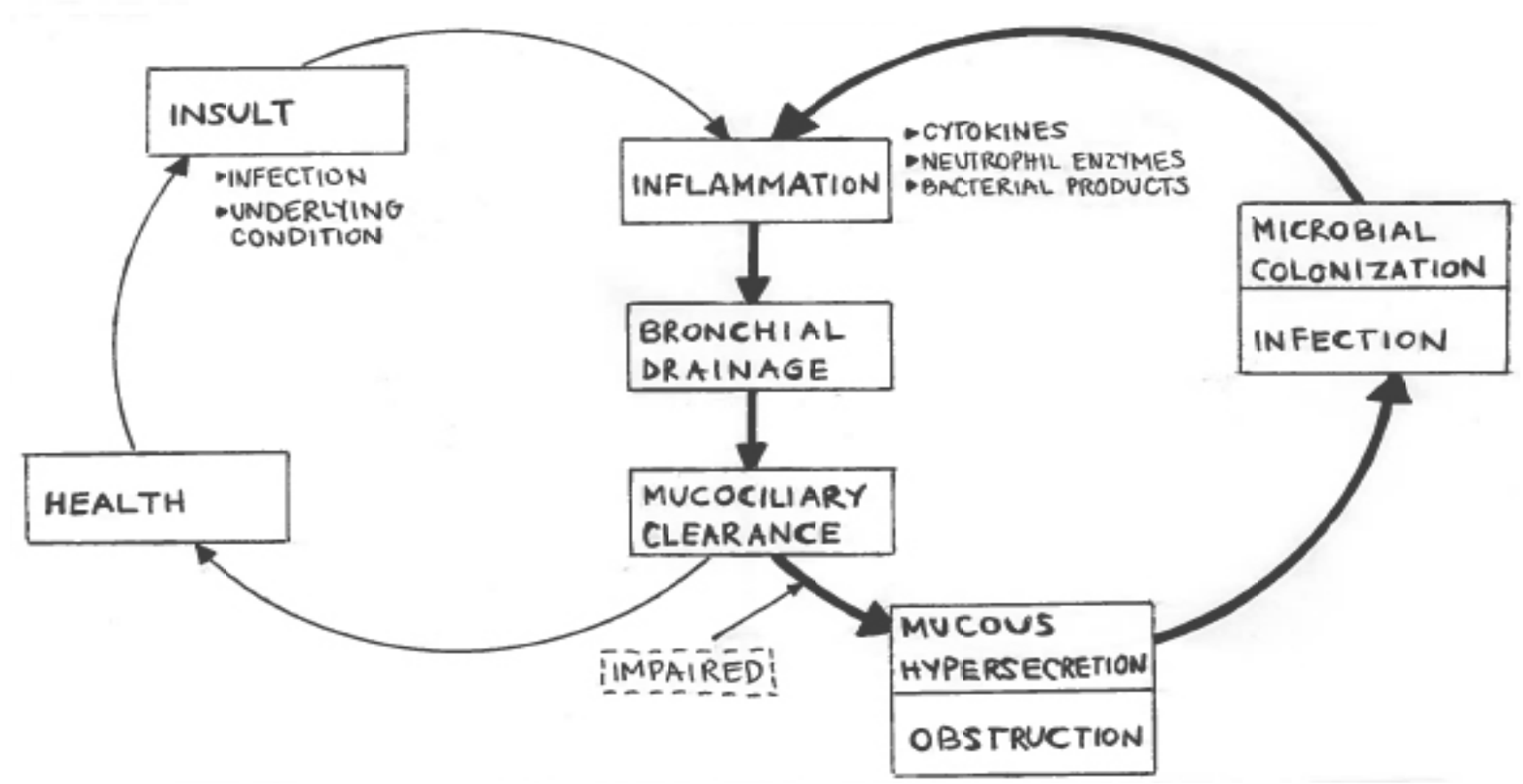

Figure I. Graphical explanation of the vicious cycle theory which renders damaged lung epithelium susceptible to further infections. http://www.chirurgiatoracica.org/images/bronch4.gif 
Microorganisms produce pigments, proteases, and other toxins that injure the respiratory epithelium, which induce an inflammatory response in the host tissue [3]. The dominant cell types involved in the inflammatory process are neutrophils, lymphocytes, and macrophages. Neutrophils are the most abundant cell type in the bronchial lumen. They play an important role in the tissue damage in bronchiectasis by releasing mediators (including inflammatory cytokines and proteases), which destroy the bronchial elastin and other supporting lung structure, leading to permanent dilatation of the bronchi. Neutrophils also alter the function of the cilial epithelium, leading to changes in cilia beat frequency and mucous gland hypersecretion. Both processes compromise mucociliary clearance [8]. This compromise leads to a further reduction in natural defense mechanisms against the invading pathogen, leaving the lung tissue susceptible to further colonization and growth of bacteria. Even if the cause is non-infectious, the changes in airway structure contribute to a pooling of mucous [8], which provokes this vicious cycle of infection and inflammation. The ultimate consequence is progressive airway damage and recurrent infections.

Primary ciliary disorder, $\mathrm{CF}$, and panhypogammaglobulinemia cause impairment of the pulmonary defense mechanism, leading to a reduced clearance of the microbial agent. Primary ciliary dyskinesia is causative of bronchiectasis in $5-10 \%$ of the disease. Clinically, the patients show recurrent respiratory infections, including sinusitis and otitis media. As the sperm motility is usually also affected, respiratory symptoms are often accompanied by impotence. Kartagener syndrome is another manifestation of primary ciliary dyskinesia. It manifests clinically as situs inversus with bronchiectasis and sinusitis [8].

\section{Non-infectious causes}

Less commonly, bronchiectasis is due to noninfectious causes. Endobronchial obstruction that leads to a dilation of the bronchi may be due to slowly growing endobronchial neoplasm (carcinoid tumors), or foreign body aspiration. These include toxic gas inhalation through, for example, ammonia. Aspiration of acidic gastric contents, as may result from a gastro-esophageal reflux disease, can also lead to bronchiectasis. Alpha 1 antitrypsin deficiency can also induce early development of panacinar emphysema or bronchiectasis. Congenital bronchiectasis can be due to primary weakness in the airways. These include the Mounier-Kuhn Syndrome (congenital tracheobronchiomegaly), the WilliamsCampbell Syndrome (absence of cartilaginous rings in the segmental and subsegmental generations of bronchi), Marfan Syndrome and relapsing polychondritis [1].

\section{Clinical manifestations}

Bronchiectasis should always be suspected in recurring purulent respiratory tract infections [3]. The most striking symptoms of bronchiectasis are persistent cough and constant production of thick, tenacious sputum [1]. Other symptoms may include halitosis (possibly as a consequence of the constant sputum production) and general debility. If the patient is suffering from an acute episode of pneumonia, pleuritic pain may indicate the infectious spread to the pleura.

Physical signs may be present on one or both sides, and appear according to the amount of secretions inside the bronchus. If the bronchiectatic airways are clear and there is no lobar collapse, physical signs may be absent. If a large amount of sputum is present, coarse crackles may be heard over the specific area of the lung. Up to $50 \%$ of the patients may present with hemoptysis [9] due to bleeding of friable and inflamed airway mucosa, which is an important complication of bronchiectasis.

\section{Diagnosis}

If the clinical presentation raises suspicion of bronchiectasis, an HRCT scan is the golden standard diagnostic procedure. Plain radiography may indicate bronchiectasis only in advanced stages, although the presence of characteristic "tram tracks" shows a persistent dilation of the airways. An HRCT scan depicts airway dilation as the parallel "tram tracks" as well as the "signet ring sign", bronchial wall thickening, mucopurulent secretions and lack of bronchial tapering. The lumen may be filled with secretions with an opaque tubular or branched tubular structure.

\section{Treatment}

The treatment of bronchiectasis remains a challenge and there are several different approaches as to which one is the best. The treatment options may be classified according to their pharmacological essence: in addition to the non-pharmacological treatment options, which describe bronchopulmonary hygiene and physical therapy, the pharmacological pillars dominate the field of treatment for bronchiectasis. These include antibiotics, anti-inflammatory agents, mucolytic agents, and bronchodilators.

Although most patients with bronchiectasis require a multitude of treatment options, there is no standard formula to rely on. The patients are rather treated in a "trial and error" approach, according to their designated needs. This may be due to the complex nature of the disease with many underlying etiologies. If a patient is diagnosed with bronchiectasis, there is a row of recommendations to prevent an exacerbation of the disease.

To prevent the occurrence of the disease, smoking cessation is a priority for all pulmonary patients. Exposure to environmental tobacco smoke increases susceptibility to respiratory infections causes adverse respiratory health outcomes and increases coughing illnesses [10]. Vaccination according to the national schedule is also a part of the management scheme in susceptible patients. 


\section{Non-pharmacological treatment}

Bronchopulmonary hygiene consists of nonpharmacological therapies directed towards mobilizing and eliminating inflammatory secretions from the tracheobronchial tree and from the paranasal sinuses. This is considered the mainstay of therapy in bronchiectasis, and although evidence base is lacking, clinical practice reflects their regular prescription and use [11]. This may be achieved by physical therapy, teaching patients lifestyle changes, evaluating the option of supplementary oxygen or considering surgery.

\section{Pharmacological treatment}

The pharmacological treatment mainly consists of antibiotics, anti-inflammatories and mucolytic agents. As bronchiectasis is a chronic disease, the patient may need psycho-education on the permanent course of the treatment plan, which should be made by a multidisciplinary team. It is important to keep side effects of chronic medication in mind and emphasis should be placed on maintaining a stable quality of life [12].

\section{Antibiotics}

The main therapeutic goal of antibiotics is to decrease the bacterial load and disrupt the infectioninflammation vicious cycle. Empiric coverage (Amoxicillin, Trimethoprim-Sulfamethoxazole, Levofloxacin) are given initially but should be guided by microbiological results. The BTS suggests the length of the antimicrobial treatment to be of 14 days or longer.

Macrolide antibiotics (especially Erythromycin and Azithromycin) have both antibacterial and antiinflammatory properties. They have excellent tissue penetration and antimicrobial activity, mainly against gram-positive cocci and intracellular pathogens [13]. Although this option might seem quite attractive, effects must be balanced with considerable expense and potential production of drug resistance: increased macrolideresistance amongst respiratory bacterial pathogens and oral streptococci have been observed [14]. This is an important concern, especially on the public health level.

The British Thoracic Society guidelines suggest long-term oral antibiotics for patients with $\geq 3$ exacerbations per year or those chronically colonized with Pseudomonas aeruginosa [15].

\section{Anti-inflammatory therapy}

The goal of the anti-inflammatory therapy is to reduce symptoms and to limit the progression of disease and decline in lung function. Options are non-steroidal antiinflammatory drugs (NSAIDs) and inhaled corticosteroids, and they are used preferably if reversible bronchospasm with airway obstruction suggesting co-existent asthma or COPD is present. Unfortunately, studies have not yet shown an improvement in lung function due to antiinflammatories - although they did demonstrate an impact on the quality of life, reducing the 24-h sputum volume and the inflammatory markers [16].

\section{Mucous-Mobilizing agents}

Effective clearance of mucus from the airways is one of the most important treatment modalities that can be instituted in patients with bronchiectasis [17]. An overview of the treatments available is summarized by the Translational Respiratory Medicine magazine in the table below [18]. There are no randomized controlled trials of anticholinergics in the treatment of acute or stable bronchiectasis. Some anticholinergic agents such as atropine and glycopyrrolate slow mucociliary transport and predispose to further mucous stasis.

\section{Treating exacerbations}

According to the guideline for the management of patients with bronchiectasis by the British Thoracic Society, 14 days of treatment with antibiotic therapy guided by previous sputum microbiology is recommended [15]. Unfortunately, this guideline is outdated by 2010, there is a great lack of prospective data on the management of bronchiectasis exacerbations (Table II). 
Table II. Algorithm of the management in bronchiectasis

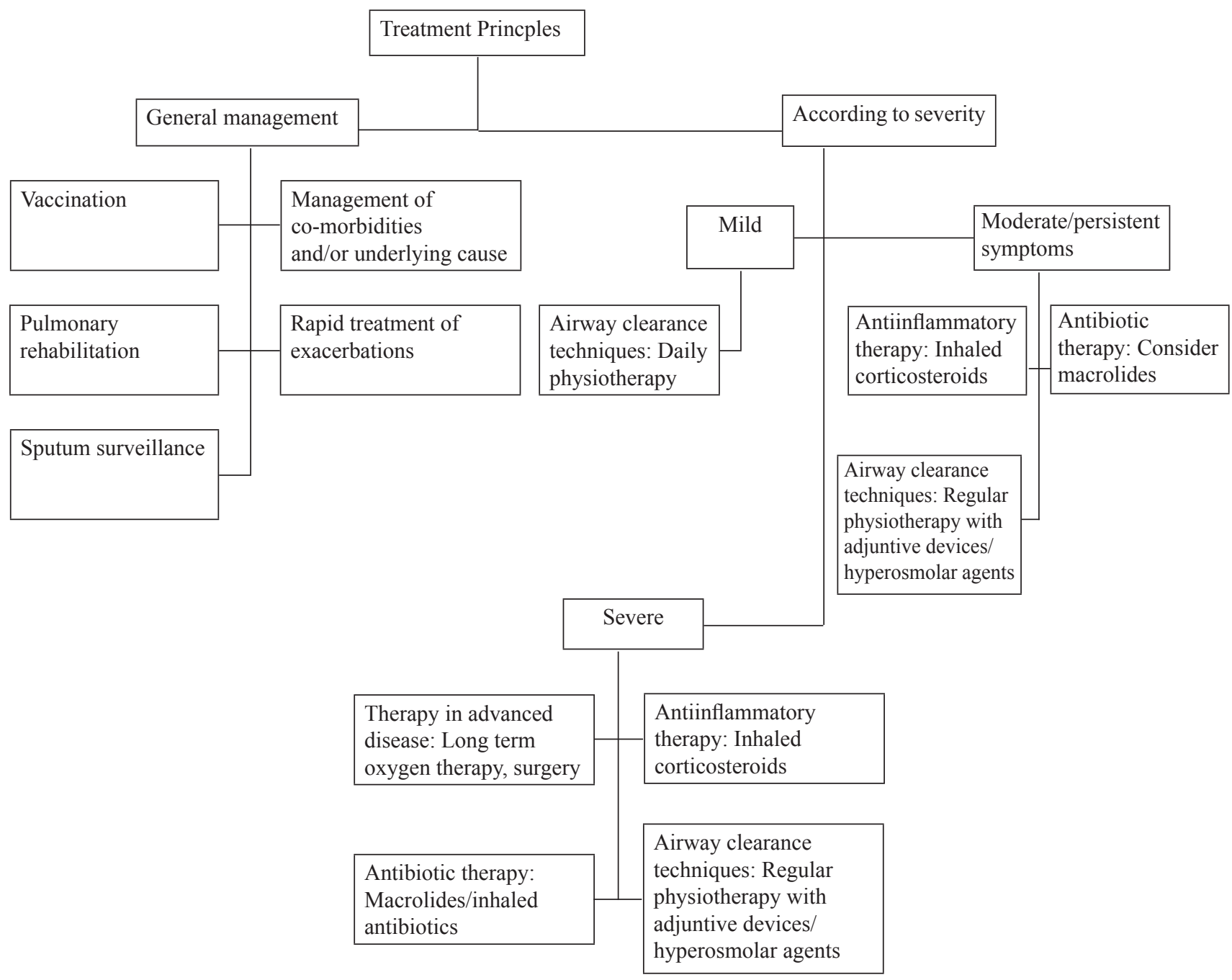

\section{Prognosis}

Early detection and clinical diagnosis to initiate a quick and effective treatment plan are essential to prognostic outcomes in bronchiectasis. The available evidence suggests that in children with bronchiectasis, early diagnosis and specialist multi-disciplinary management can stabilize the prognosis of the disease. A better understanding of management will not only improve short- and long-term outcomes in children but also prevent severe bronchiectasis in adults [10].

Adults with newly diagnosed bronchiectasis, but with symptoms dating back to childhood, had significantly more exacerbations, lower lung function, and worse HRCT scan scores than those whose symptoms began later in life. Additionally, exacerbation frequency can potentially be reduced, simultaneously preserving lung function when children receive specialist and multi-disciplinary care at an early stage of the disease [19].

\section{References}

1. Theodore A. Kotchen. Harrisons Principle of Internal Medicine 18th Edition . Harrisons Principles of Internal Medicine. 2015. pp 2042, 2048.

2. Olveira C, Padilla A, Martínez-García MÁ, de la Rosa D, Girón RM, Vendrell M, et al. Etiology of Bronchiectasis in a Cohort of 2047 Patients. An Analysis of the Spanish Historical Bronchiectasis Registry. Arch Bronconeumol. 2017;53:366-374.

3. Dr. Hamidi, Hidayatullah and Prof. Gaillard, Frank. "Bronchiectasis". Radiopaedia.org Available from: https:// radiopaedia.org/articles/bronchiectasis

4. Redondo M, Keyt H, Dhar R, Chalmers JD. Global impact of bronchiectasis and cystic fibrosis. Breathe (Sheff). 2016;12:222235.

5. King PT. The pathophysiology of bronchiectasis. Int J Chron Obstruct Pulmon Dis. 2009;4:411-419.

6. Langendorfer B. Alternatives to percussion and postural drainage. A review of mucus clearance therapies: percussion and postural drainage, autogenic drainage, positive expiratory pressure, flutter valve, intrapulmonary percussive ventilation, 
and high-frequency chest compression with the ThAIRapy Vest. J Cardiopulm Rehabil. 1998;18:283-289.

7. Chang AB, Redding GJ. Bronchiectasis and chronic suppurative lung disease. Kendig Chernick's Disord Respir Tract Child. 2012; p 473-88.

8. Nomellini V, Chen H. Murray and Nadel's Textbook of Respiratory Medicine [Internet]. Vol. 173, Journal of Surgical Research. 2012. p 45.

9. Davidson SS. Davidson 's Medicine. Davidson's principles and practice of medicine. 2010. p. 498-499.

10. Kanoh S, Rubin BK. Mechanisms of action and clinical application of macrolides as immunomodulatory medications. Clin Microbiol Rev. 2010;23:590-615.

11. Lee AL, Button BM, Tannenbaum EL. Airway-Clearance Techniques in Children and Adolescents with Chronic Suppurative Lung Disease and Bronchiectasis. Front Pediatr. 2017;5:2.

12. Chalmers JD, Sethi S. Raising awareness of bronchiectasis in primary care: overview of diagnosis and management strategies in adults. NPJ Prim Care Respir Med. 2017;27(1):18.
13. Guran T, Ersu R, Karadag B, Karakoc F, Demirel GY, Hekim $\mathrm{N}$, et al. Withdrawal of inhaled steroids in children with noncystic fibrosis bronchiectasis. J Clin Pharm Ther. 2008;33:603611.

14. Taylor SP, Sellers E, Taylor BT. Azithromycin for the Prevention of COPD Exacerbations: The Good, Bad, and Ugly. Am J Med. 2015;128:1362.e1-e6.

15. Pasteur MC, Bilton D, Hill AT; British Thoracic Society Bronchiectasis non-CF Guideline Group. British Thoracic Society guideline for non-CF bronchiectasis. Thorax. 2010;65 Suppl 1:i1i58.

16. Chalmers JD, Aliberti S, Blasi F. Management of bronchiectasis in adults. Eur Respir J. 2015;45:1446-1462.

17. Feldman C. Bronchiectasis: new approaches to diagnosis and management. Clin Chest Med. 2011;32:535-546.

18. Rubin BK. Secretion properties, clearance, and therapy in airway disease. Transl Respir Med. 2014;2:6.

19. Chalmers JD. Bronchiectasis: Phenotyping a Complex Disease. COPD. 2017;14(sup1):S12-S18. 